\title{
CUSTOM MADE C18 COLUMNS FOR THE HYDROPHOBIC CHROMATOGRAPHY OF CEPHALOSPORIN C DERIVATIVES
}

\author{
E. Roderick White and Margaret Fox \\ Analytical \& Physical Chemistry Department, Research \& Development, \\ Smith Kline \& French Laboratories, 1500 Spring Garden Street, P.O. Box 7929, Philadelphia, \\ Pennsylvania 19101 U. S. A.
}

(Received for publication April 20, 1982)

\begin{abstract}
"Hydrophobic chromatography", which is a variation of reverse phase chromatography, is applicable to the analysis of cephalosporin $\mathrm{C}$ derivatives, especially in fermentation broths. Unfortunately, there are no commercial C18 columns which are entirely suitable for this class of compounds. For this reason $\mathrm{C} 18$ columns were prepared by an in-situ bonding technique and were optimally designed for cephalosporin $\mathrm{C}$ derivatives. Mono-, di- and trifunctional octadecyl bonding agents were used with $10 \mu \mathrm{m}$ silica of both $60 \AA$ and $100 \AA$ pore diameter. The best results were obtained with the difunctional agent, methyloctadecyldichlorosilane, and $100 \AA$ silica. "Endcapping" of residual silanol groups with a trimethylsilylation agent was optional, since good results were obtained with both a plain C18 column and one that was "endcapped".
\end{abstract}

"Hydrophobic chromatography" is a variation of the reverse phase technique in which a neat (i.e., no organic component) aqueous solvent is used as the mobile phase. This mode of chromatography is very useful for the analysis of polar ionic compounds. In particular, it is well suited for the separation and quantitation of cephalosporin $\mathrm{C}$ derivatives, especially in fermentation broths.

In a previous paper ${ }^{1)}$ we described hydrophobic conditions for the separation of cephalosporin $\mathrm{C}$ using a large particle $(37 \sim 50 \mu \mathrm{m})$, medium efficiency $\mathrm{C} 18$ column. In a second paper ${ }^{2)}$ we presented an improved method for the analysis of cephalosporin $\mathrm{C}$ derivatives using a small particle $(10 \mu \mathrm{m})$, high efficiency column prepared in-house by chemically bonding octadecyltrichlorosilane to silica by an insitu technique. An in-situ column was used because no commercially available column was completely satisfactory for use in the hydrophobic mode. Poor efficiencies and broad asymmetrical peaks are often obtained with commercial columns. In addition, both retention and resolution properties are inadequate, especially with early eluting compounds such as deacetylcephalosporin $\mathrm{C}$ and deacetoxycephalosporin $\mathrm{C}$.

Cephalosporin $\mathrm{C}$ derivatives have been separated by $\mathrm{us}^{3)}$ and others ${ }^{4)}$ with reverse phase ion-pair chromatography. The principal advantage of this approach is that early eluting compounds such as deacetylcephalosporin $\mathrm{C}$ and deacetoxycephalosporin $\mathrm{C}$ are retained on the column long enough to be well separated from interfering substances in fermentation broths. However, with a properly prepared in-situ column as described in this paper, good separations can be obtained without the need for ion-pairing.

The work ${ }^{2)}$ that we reported previously on the hydrophobic chromatography of cephalosporin $\mathrm{C}$ derivatives employed an in-situ C18 column. However, at that time there was no attempt made to discover the optimum conditions for producing these in-situ columns. This paper gives the results of a study that was carried out on the preparation of $\mathrm{C} 18$ columns especially suited for the hydrophobic 
chromatography of cephalosporin C derivatives. A variety of commerical columns were evaluated, but none was found to be suitable for reasons stated earlier. Since commercial columns were not suitable, a series of in-situ C18 columns were prepared and evaluated. The columns were prepared from SI 60 and SI 100 silica using mono, di- and trifunctional octadecyl bonding agents with and without endcapping of residual silanol groups. The columns were evaluated with respect to efficiency, peak symmetry and general ability to separate cephalosporin $\mathrm{C}$ derivatives in fermentation broths. The best results were obtained with $100 \AA$ silica, a difunctional bonding agent and endcapping was optional. These in-situ $\mathrm{C} 18$ columns have been used for over five years in a cephalosporin $\mathrm{C}$ fermentation program.

\section{Experimental}

Apparatus

The liquid chromatograph used in this work was self-built and consisted of a pump (Model 26980-4, Haskel Engineering and Supply Co., Burbank, California), UV detector (Model No. 153, Altex Scientific, Inc., Berkeley, California) and sample valve (Model No. 70-10, Rheodyne, Berkeley, California). The holding tube for the silane solutions was constructed from 316 s.s. tubing with dimensions $90 \mathrm{~cm} \times$ $6.3 \mathrm{~mm}$ (o.d.) $\times 4.6 \mathrm{~mm}$ (i.d.). A minipump (Model 396-57, Milton Roy Company, Riviera Beach, Florida) was used to pump the silane solution out of the holding tube and onto the silica columns where bonding reactions occur.

\section{Reagents}

Acetonitrile, heptane, isopropanol, methanol, methylene chloride and toluene were obtained from Burdick and Jackson Laboratories, Inc., Muskegon, Michigan. Octadecyltrichlorosilane (ODTCS), methyloctadecyldichlorosilane (MODDCS), and dimethyloctadecylchlorosilane (DMODCS) were obtained from Petrarch Systems, Inc., Levittown, Pennsylvania. Bis(trimethylsilyl)trifluoroacetamide (BSTFA) plus $1 \%$ trimethylchlorosilane (TMCS) was obtained from Regis Chemical Company, Morton Grove, Illinois. The water used in the mobile phase was deionized and distilled. DL- $\alpha$-Methylbenzyl alcohol, benzyl alcohol and cinnamyl alcohol were obtained from Chem Service, Inc., West Chester, Pennsylvania.

\section{Materials}

The cephalosporins used in this study were obtained from the Investigational Products Laboratory of Smith Kline \& French Laboratories. The fermentation broths were provided by CharLes PAN. Deacetylcephalosporin $\mathrm{C}$ and deacetoxycephalosporin $\mathrm{C}$ were generously provided by Dr. NORBERT Neuss (Lilly Research Laboratories, Indianapolis, Indiana).

Column Packings

$10 \mu \mathrm{m}$ Lichrosorb SI 60 and SI 100 packings were obtained from MCB Manufacturing Chemists, Inc., Associate of E. Merck, Cincinnati, Ohio.

Commercial Columns

The Zorbax-ODS and C8 columns were obtained from Dupont Instruments, Wilmington, Delaware.

The Partisil ODS, ODS-2 and ODS-3 columns were obtained from Whatman, Inc., Clifton, New Jersey.

The $\mu$ Bondapak C18 column was obtained from Waters Associates, Milford, Connecticut.

\section{In-Situ Columns}

The in-situ columns were made from silica columns which were prepared in our laboratories by a balanced density slurry packing procedure ${ }^{5)}$. The silica columns were tested with a mixture of DL- $\alpha$ methylbenzyl alcohol, benzyl alcohol and cinnamyl alcohol and a mobile phase of heptane - methylene chloride - isopropanol (900: 94: 6).

The procedure used to prepare the in-situ bonded columns is a modification of that used by GILPIN ${ }^{6)}$. However, instead of carrying out the bonding reaction in the presence of a controlled amount of water on 
the surface of the silica, anhydrous conditions were maintained in this work. This modification was made in order to prevent polymerization and thus obtain monomolecular coverage of the silica surface.

The step-by-step procedure used for carrying out the in-situ bonding reactions is as follows:

1) $500 \mathrm{ml}$ of dry toluene $(<0.01 \%$ water) was pumped through the column at a rate of $5 \mathrm{ml} /$ minute.

2) The column was disconnected from the pump and a $90 \mathrm{~cm} \times 6.3 \mathrm{~mm}$ (o.d.) $\times 4.6 \mathrm{~mm}$ (i.d.) stainless steel holding tube filled with $15 \mathrm{ml}$ of a $10 \%$ solution of the appropriate silane in dry toluene was connected between the pump and the column.

3) The silane solution was pumped through the column at a rate of $0.3 \mathrm{ml} / \mathrm{minute}$ until $40 \mathrm{ml}$ of solution was collected, followed by $500 \mathrm{ml}$ of dry toluene at a rate of $5 \mathrm{ml} / \mathrm{minute}$. This volume of dry toluene was required to purge the column of unreacted reagent.

4) If endcapping was desired, steps 2 and 3 were repeated with a solution of $10 \%$ Regisil (BSTFA+ $1 \%$ TMCS) as the bonding agent.

5) The column was rinsed with $200 \mathrm{ml}$ of acetonitrile at a rate of $5 \mathrm{ml} / \mathrm{minute}$. This volume of acetonitrile was required to purge the column of toluene and thus make it compatible with aqueous solvents used in reverse phase chromatography.

In the reverse phase mode the columns were tested with toluene as a test substance and a mobile phase of methanol - water (60:40).

In the hydrophobic mode the columns were evaluated with cephalosporin $\mathrm{C}$ and a mobile phase as described in the next section.

Hydrophobic Mobile Phase

The hydrophobic mobile phase used in this work was water containing potassium dihydrogen phosphate as an ionic modifier. However, similar results can be obtained with either sodium or ammonium dihydrogen phosphate. In general, the capacity factor $\left(\mathrm{k}^{\prime}\right)$ for cephalosporin $\mathrm{C}$ increases with increasing phosphate concentration. All of the separations reported here were carried out with an aqueous mobile phase containing an arbitrarily set concentration of $0.03 \%$ potassium dihydrogen phosphate ( $\mathrm{pH} 4.5$ ).

\section{Results and Discussion}

Both the commercial and in-situ columns were evaluated with respect to efficiency or number of theoretical plates, $\mathrm{N}$, retention ability or capacity factor, $\mathrm{k}^{\prime}$, and peak asymmetry, $\mathrm{A}_{\mathrm{s}}$. The definitions of these terms are given in Fig. 1.

\section{Commercial Columns}

One of the first columns tried for the separation of cephalosporin C derivatives was a Zorbax ODS column. The separation of a synthetic mix containing deacetylcephalosporin $\mathrm{C}$, deacetoxycephalosporin $\mathrm{C}$ and cephalosporin $\mathrm{C}$ is shown in Fig.

2. When tested with toluene, this column was very efficient ( $\sim 14,000$ theoretical plates). However, the efficiency is very poor ( $\sim 650$ theoretical plates) for cephalosporin C. In addition, there is insufficient separation of deacetylcephalosporin $\mathrm{C}$ from the solvent front. Clearly, this column is unsuitable for the separation of cephalosporin $\mathrm{C}$ derivatives. It is a heavily loaded $(\sim 16 \%$ carbon) $\mathrm{C} 18$ column and is probably too hydro-

Fig. 1. Definitions of chromatography parameters.

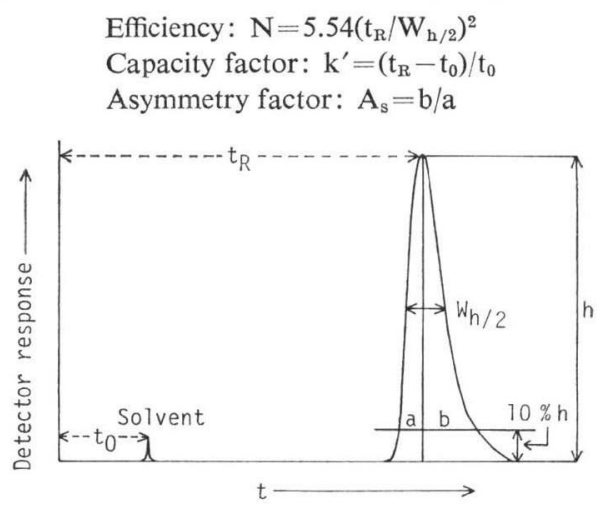


Fig. 2. Column: $25 \mathrm{~cm} \times 4.6 \mathrm{~mm}$ (i.d.), Zorbax ODS: mobile phase, $0.03 \%$ potassium dihydrogen phosphate; pressure, $70 \mathrm{~kg} / \mathrm{cm}^{2}$; flow, $1.1 \mathrm{ml} /$ minute; detector, UV (254 $\mathrm{nm})$; sensitivity, 0.16 AUFS; sample, synthetic mix in water; sample size, $20 \mu \mathrm{l}$.

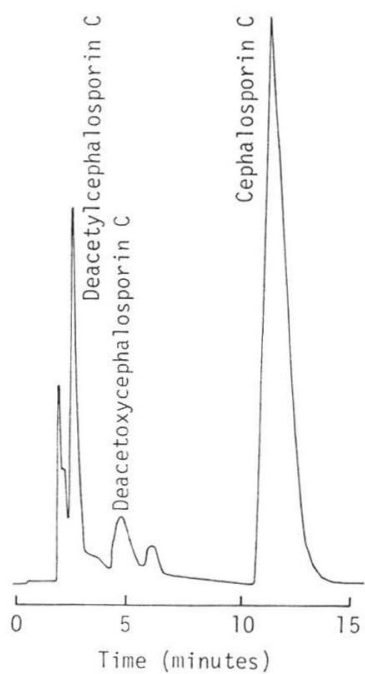

Fig. 3. Column: $25 \mathrm{~cm} \times 4.6 \mathrm{~mm}$ (i. d.), Partisil ODS; mobile phase, $0.03 \%$ potassium dihydrogen phosphate; pressure, $56 \mathrm{~kg} / \mathrm{cm}^{2}$; flow, $1.0 \mathrm{ml} /$ minute; detector, UV $(254 \mathrm{~nm})$; sensitivity 0.32 AUFS; sample, synthetic mix in water; sample size, $20 \mu 1$.

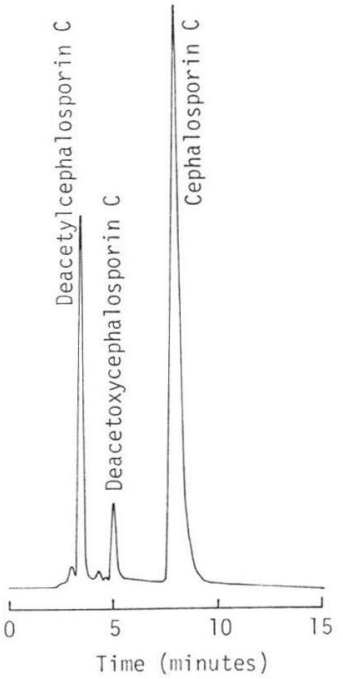

Fig. 4. Column: $25 \mathrm{~cm} \times 4.6 \mathrm{~mm}$ (i. d.), Partisil ODS-2; mobile phase, $0.03 \%$ potassium dihydrogen phosphate; pressure, $70 \mathrm{~kg} / \mathrm{cm}^{2}$; flow, $1.1 \mathrm{ml} /$ minute; detector, UV $(254 \mathrm{~nm})$; sensitivity, 0.04 AUFS; sample, fermentation broth; sample size, $20 \mu 1$.

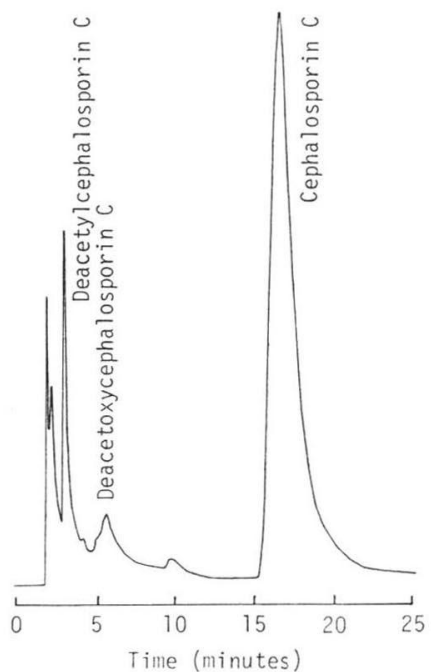

phobic for the highly polar cephalosporins.

Three C1 8 columns obtained from Whatman were evaluated. Separations on Partisil ODS, ODS-2 and ODS-3 are shown in Figs. 3 to 5 . The efficiency of the ODS column is reasonable $(\sim 1,200$ theoretical plates), but deacetylcephalosporin $\mathrm{C}$ is not sufficiently separated from the solvent front and the capacity factors are too small for all three compounds. Fig. 4 shows that the ODS- 2 column is also unsuitable. The efficiency is low ( $\sim 500$ theoretical plates), the peaks tail and deacetylcephalosporin $\mathrm{C}$ is not separated sufficiently from early eluting components. ODS- 2 is a heavily loaded $(\sim 17 \%$ carbon) "polymeric" type C18 column which is also probably too hydrophobic for cephalosporins. As shown in Fig. 5, the ODS-3 column gives badly tailing peaks and the capacity factors, especially for cephalosporin $\mathrm{C}$, are much too large for practical use. The ODS-3 column is a "monomeric" type column, but is exhaustively covered with C18 groups ( $~ 95 \%$ coverage). Obviously, this column is also too hydrophobic for cephalosporins.

The separation obtained on a Waters $\mu$ Bondapak C18 column is shown in Fig. 6. While deacetylcephalosporin $\mathrm{C}$ and deacetoxycephalosporin $\mathrm{C}$ are well separated, cephalosporin $\mathrm{C}$ tails badly. It is interesting to compare this separation with that obtained on the ODS-3 column, since Whatman claims that this column has selectivity similar to a $\mu$ Bondapak C18 column. Obviously, the selectivities of the two columns are very different with cephalosporin $\mathrm{C}$ derivatives.

The only C8 column evaluated in this work was a Zorbax C8, since Dupont claims that this column can be substituted for a $\mu$ Bondapak C18 column for some applications. The separation obtained on the Zorbax $\mathrm{C} 8$ column is shown in Fig. 7. Clearly, it is unsuitable for cephalosporin $\mathrm{C}$ derivatives because 
Fig. 5. Column: $25 \mathrm{~cm} \times 4.6 \mathrm{~mm}$ (i.d.), Partisil ODS- 3 ; mobile phase, $0.03 \%$ potassium dihydrogen phosphate; pressure, $105 \mathrm{~kg} / \mathrm{cm}^{2}$; flow, $1.2 \mathrm{ml} /$ minute; detector, UV (254 nm); sensitivity, 0.08 AUFS; sample, synthetic mix in water; sample size, $20 \mu 1$.

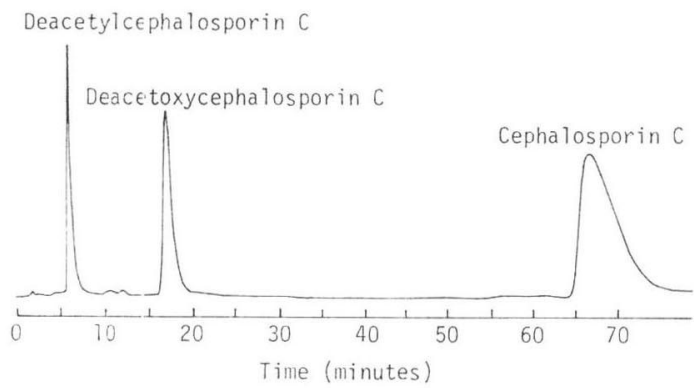

Fig. 6. Column: $30 \mathrm{~cm} \times 3.9 \mathrm{~mm}$ (i. d.), $\mu$ Bondapak C18; mobile phase, $0.03 \%$ potassium dihydrogen phosphate; pressure, $62 \mathrm{~kg} / \mathrm{cm}^{2}$; flow, $1.0 \mathrm{ml} /$ minute; detector, UV (254 nm); sensitivity, 0.16 AUFS; sample, synthetic mix in water; sample size, $20 \mu 1$.

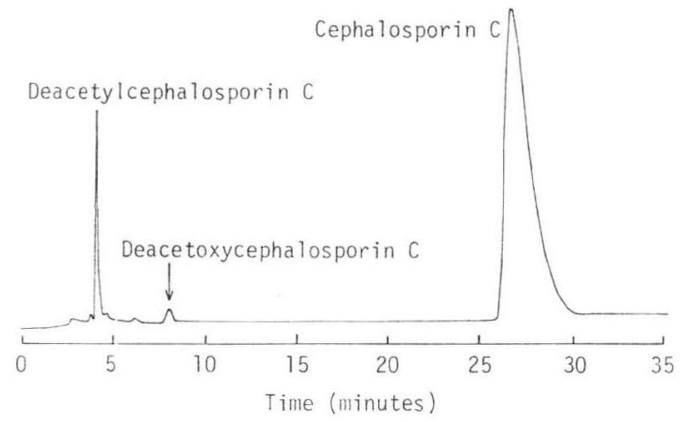

Fig. 7. Column: $25 \mathrm{~cm} \times 4.6 \mathrm{~mm}$ (i. d.), Zorbax C8; mobile phase, $0.03 \%$ potassium dihydrogen phosphate; pressure, $70 \mathrm{~kg} / \mathrm{cm}^{2}$; flow, $1.0 \mathrm{ml} /$ minute; detector, UV (254 nm); sensitivity, 0.32 AUFS; sample, synthetic mix in water; sample size, $20 \mu \mathrm{l}$.

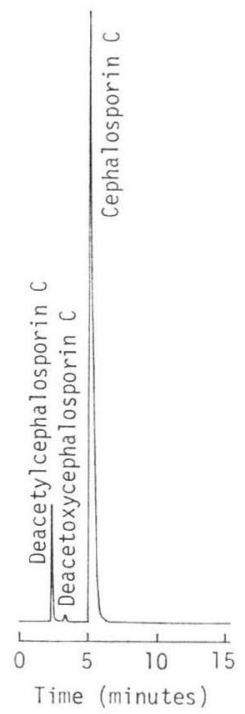

of a lack of sufficient retention. In addition, if this separation is compared with that obtained on the $\mu$ Bondapak C18 column (Fig. 6), it is obvious that the Zorbax C8 column behaves much differently than the $\mu$ Bondapak C18 with cephalosporin $\mathrm{C}$ derivatives.

A summary of determined physical parameters for all of the commercial columns studied is given in Table 1. Values of $\mathbf{N}$ and $\mathbf{A}_{\mathrm{s}}$, both calculated from the cephalosporin $\mathrm{C}$ peak, as well as $\mathrm{k}^{\prime}$ values for all three cephalosporin $\mathrm{C}$ derivatives are tabulated. All of the columns have a minimum of 5,000 theoretical plates when tested with toluene. However the real efficiencies as determined from cephalosporin $\mathrm{C}$ are much lower than this value. The two columns with the lowest efficiencies, Zorbax ODS and Partisil ODS-2, are very heavily loaded and are too hydrophobic to be useful for the separation of cephalosporin $\mathrm{C}$ derivatives. Excessive peak tailing is observed on the ODS-2, ODS-3 and $\mu$ Bondapak $\mathrm{C} 18$ columns as indicated by the large values for $\mathrm{A}_{\mathbf{s}}$. Columns with a value greater than two for $\mathrm{A}_{\mathrm{s}}$ are considered inadequate. The $\mathrm{k}^{\prime}$ values for the Partisil ODS and Zorbax C8 columns are too low, while they are much too large for the ODS-3 column.

In spite of the low $\mathrm{k}^{\prime}$ values, the column that comes closest to being adequate is the Partisil ODS column. The efficiency is relatively high ( $\sim 1,200$ theoretical plates) and the $\mathrm{A}_{\mathrm{s}}$ value (2.1) is borderline but acceptable. This column is only about 50\% covered with $\mathrm{C} 18$ groups and has about $50 \%$ exposed silanol groups. It appears that the highly polar cephalosporin $\mathrm{C}$ derivatives need to interact with some of the exposed silanol groups and, as a result, reasonably high efficiencies and symmetrical peaks are obtained with this column. However, this column cannot be used, since the $\mathrm{k}^{\prime}$ values are 
Table 1. Physical parameters of commercial columns.

\begin{tabular}{lrrccc}
\hline \multirow{2}{*}{ Column } & \multirow{2}{*}{$\mathrm{N}$} & \multirow{2}{*}{$\mathrm{A}_{\mathrm{s}}$} & \multicolumn{3}{c}{${\text { Capacity factors, } \mathrm{k}^{\prime}}^{n}$} \\
\cline { 5 - 6 } & & & Deacetyl & Deacetoxy & Ceph C \\
\hline Dupont Zorbax ODS & 659 & 1.4 & 0.5 & 1.5 & 5 \\
Whatman Partisil PXS 10/25 ODS & 1348 & 2.1 & 0.4 & 1.0 & 2.1 \\
Whatman Partisil PXS 10/25 ODS-2 & 522 & 2.8 & 0.6 & 1.9 & 7.3 \\
Whatman Partisil PXS 10/25 ODS-3 & 1209 & 3.3 & 2.3 & 8.4 & 32 \\
Waters $\mu$ Bondapak C18 & 1370 & 3.7 & 0.9 & 2.6 & 11 \\
Dupont Zorbax C8 & 1047 & 1.6 & 0.3 & 0.8 & 1.8 \\
\hline
\end{tabular}

much too small.

\section{In-Situ Columns}

Since none of the commercial columns were completely satisfactory, a series of C18 columns were prepared by the $i n$-situ technique in an attempt to obtain a column uniquely suited for the hydrophobic chromatography of cephalosporin $\mathrm{C}$ derivatives.

A summary of the measured parameters for the in-situ columns is given in Table 2. This table is similar to Table 1, except that an additional parameter is included. The ratio, $\left(\mathrm{N}_{\mathrm{after}} / \mathrm{N}_{\text {before }}\right)$ is an approximate measure of the effectiveness of the bonding reaction where $\left(\mathrm{N}_{\text {before }}\right)$ is the plate number of the silica column and $\left(\mathrm{N}_{\mathrm{after}}\right)$ is the plate number of the corresponding C18 column as calculated from the toluene peak. The various in-situ columns are described in the table in terms of the bonding agent and the silica used in preparing them.

The efficiencies of the silica columns were in the range of 6,000 to 7,000 theoretical plates and all of the uncapped columns, but one, gave similar efficiencies in the reverse phase mode $\left(\mathrm{N}_{\mathrm{after}} / \mathrm{N}_{\text {before }}\right)=1$. The only exception to this was with DMODCS, SI 100 which gave a column with a value of 0.5 for $\left(\mathrm{N}_{\mathrm{after}} / \mathrm{N}_{\text {before }}\right)$. As shown in the table, about 10 to $20 \%$ efficiency is lost as a result of the endcapping step.

Table 2. Physical parameters of in-situ columns.

\begin{tabular}{|c|c|c|c|c|c|c|}
\hline \multirow{2}{*}{ Column } & \multirow{2}{*}{$\begin{array}{c}\mathrm{N}_{\text {after }} \\
\mathrm{N}_{\text {before }}\end{array}$} & \multirow{2}{*}{$\mathrm{N}$} & \multirow{2}{*}{$\mathrm{A}_{\mathrm{s}}$} & \multicolumn{3}{|c|}{ Capacity factors, $\mathrm{k}^{\prime}$} \\
\hline & & & & Deacetyl & Deacetoxy & Ceph C \\
\hline ODTCS, SI 60 & 1.0 & 995 & 2.1 & 0.4 & 1.1 & 3.3 \\
\hline ODTCS, SI 100 & 1.0 & 1200 & 1.2 & 0.4 & 0.7 & 1.7 \\
\hline ODTCS+TMS, SI 100 & 0.9 & 900 & 1.2 & 0.3 & 1.1 & 4.7 \\
\hline DMODCS, SI 60 & 1.0 & 900 & 2.2 & 0.4 & 1.1 & 4.0 \\
\hline DMODCS, SI 100 & 0.5 & 970 & 1.2 & 0.6 & 1.4 & 4.2 \\
\hline MODDCS, SI 60 & 1.0 & 1280 & 2.6 & 0.6 & 1.6 & 5.0 \\
\hline MODDCS + TMS, SI 60 & 0.9 & 900 & 2.3 & 0.6 & 1.8 & 8.3 \\
\hline MODDCS + TMS, SI 100 & 0.8 & 1400 & 1.6 & 0.8 & 2.1 & 8.6 \\
\hline MODDCS, SI 100 & 1.0 & 1300 & 1.0 & 0.6 & 1.6 & 5.3 \\
\hline
\end{tabular}

ODTCS-Octadecyltrichlorosilane

MODDCS-Methyloctadecyldichlorosilane

DMODCS-Dimethyloctadecylchlorosilane

TMS-Trimethylsilyl 
Without exception, tailing peaks are obtained for cephalosporin $\mathrm{C}$ on all columns prepared from SI 60 silica. This is evident from an inspection of the asymmetry factors, $A_{8}$, for these columns. All of the SI 60 columns have $\mathrm{A}_{\mathrm{s}}$ values greater than two, which is generally considered to be the limit for acceptable columns. A possible way to decrease peak tailing is by endcapping. However, as shown in the table, $A_{s}$ did not decrease significantly in the case of the MODDCS + TMS, SI 60 column. It appears that the tailing is an inherent characteristic of $60 \AA$ silica, perhaps indicating that $60 \AA$ is not a sufficiently large pore diameter for the bulky $\mathrm{C} 18$ chains. Because of the tailing problem SI 60 silica was considered to be unsuitable for the present application.

Columns prepared with the trifunctional bonding agent (ODTCS) were not suitable for the separation of cephalosporin $\mathrm{C}$ derivatives. The main deficiency of these columns is the lack of sufficient retentivity. As seen in the table, the $\mathrm{k}^{\prime}$ values for all of the ODTCS columns are too small. This is especially true for deacetylcephalosporin $\mathrm{C}$, which is not separated sufficiently from the solvent front. This fact is illustrated in Fig. 8 which is the separation of a synthetic mix of cephalosporins on an ODTCS, SI 100 column.

Most commercial $\mathrm{C} 18$ columns are made using the monofunctional bonding agent dimethyloctadecylchlorosilane. With this reagent monolayer coverage is guaranteed since polymerization is not possible with a monofunctional chlorosilane. The DMODCS, SI 60 column obtained with this reagent is unsatisfactory, mainly because of the tailing, but also because of the low efficiency and small $\mathrm{k}^{\prime}$ values. The DMODCS, SI 100 column is adequate but not outstanding. This column is somewhat anomalous in that a relatively high plate count is obtained for cephalosporin C (970) in spite of the fact that it is a

Fig. 8. Column: $30 \mathrm{~cm} \times 4.6 \mathrm{~mm}$ (i.d.); ODTCS, SI 100, $10 \mu$; mobile phase, $0.03 \%$ potassium dihydrogen phosphate; pressure, $42 \mathrm{~kg} / \mathrm{cm}^{2}$; flow, $1.1 \mathrm{ml} /$ minute; detector, UV (254 nm); sensitivity, 0.16 AUFS; sample, synthetic mix in water; sample size, $20 \mu 1$.

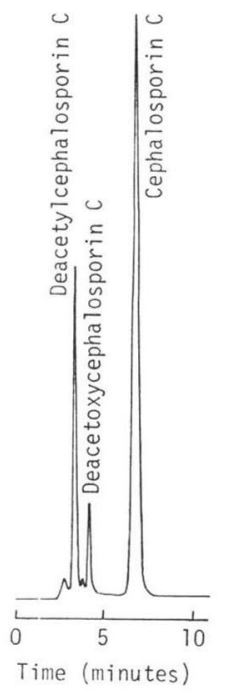

Fig. 9. Column: $30 \mathrm{~cm} \times 4.6 \mathrm{~mm}$ (i.d.), DMODCS, SI 100, $10 \mu$; mobile phase, $0.03 \%$ potassium dihydrogen phosphate; pressure, $42 \mathrm{~kg} / \mathrm{cm}^{2}$; flow $1.0 \mathrm{ml}$ / minute; detector, UV (254 nm); sensitivity 0.16 AUFS; sample, synthetic mix in water; sample size, $20 \mu 1$.

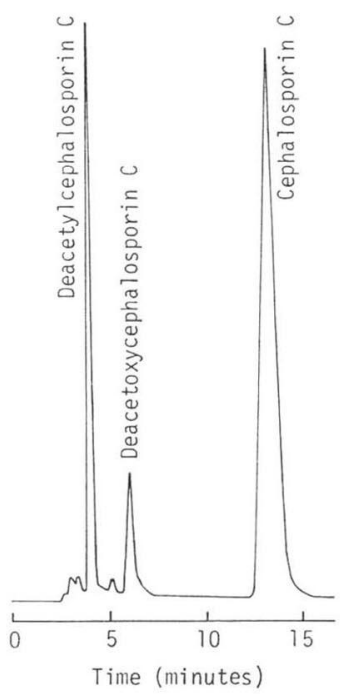

Fig. 10. Column: $30 \mathrm{~cm} \times 4.6 \mathrm{~mm}$ (i.d.), MODDCS, SI $60,10 \mu$; mobile phase $0.03 \%$ potassium dihydrogen phosphate; pressure, $35 \mathrm{~kg} / \mathrm{cm}^{2}$; flow, $1.0 \mathrm{ml} /$ minute; detector, UV (254 nm); sensitivity, 0.16 AUFS; sample, synthetic mix in water; sample size, $20 \mu 1$.

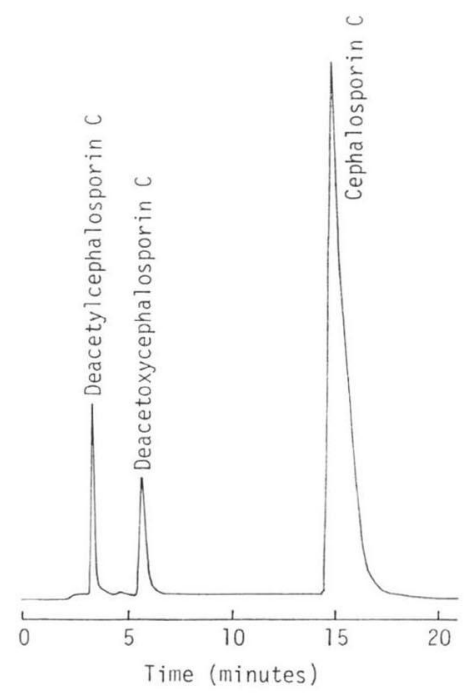


Fig. 11. Column: $30 \mathrm{~cm} \times 4.6 \mathrm{~mm}$ (i.d.), MODDCS + TMS, SI 60, $10 \mu$; mobile phase, $0.03 \%$ potassium dihydrogen phosphate; pressure, $49 \mathrm{~kg} / \mathrm{cm}^{2}$; flow, $1.0 \mathrm{ml} / \mathrm{minute;} \mathrm{detector,}$ UV (254 nm); sensitivity, 0.08 AUFS; sample, synthetic mix in water; sample size, $20 \mu 1$.

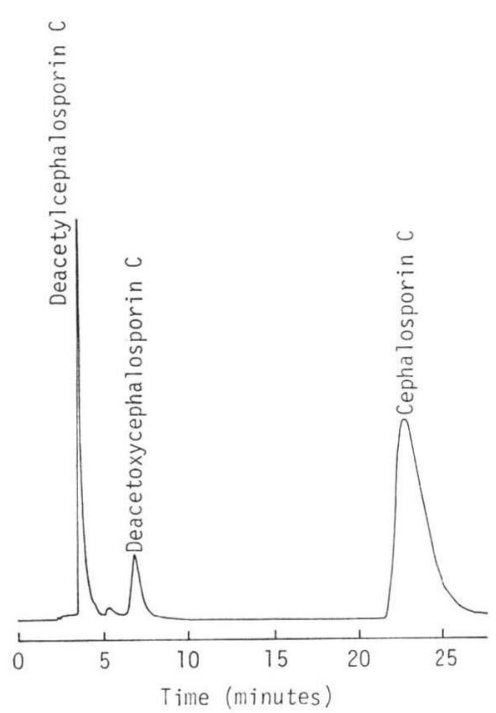

Fig. 12. Column: $30 \mathrm{~cm} \times 4.6 \mathrm{~mm}$ (i.d.); MODDCS, SI 100, $10 \mu$; mobile phase, $0.03 \%$ potassium dihydrogen phosphate; pressure, $42 \mathrm{~kg} / \mathrm{cm}^{2}$; flow, $1.0 \mathrm{ml} /$ minute; detector, UV (254 nm); sensitivity, 0.16 AUFS; sample, synthetic mix in water; sample size, $20 \mu 1$.

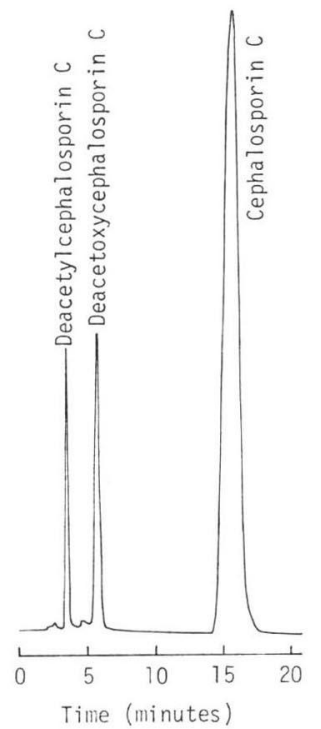

Fig. 13. Column: $30 \mathrm{~cm} \times 4.6 \mathrm{~mm}$ (i.d.), MODDCS + TMS, SI $100,10 \mu$; mobile phase, $0.03 \%$ potassium dihydrogen phosphate; pressure, $49 \mathrm{~kg} / \mathrm{cm}^{2}$; flow, $1.0 \mathrm{ml} / \mathrm{minute;} \mathrm{detector,}$ UV (254 nm); sensitivity, 0.08 AUFS; sample, fermentation broth; sample size, $20 \mu 1$.

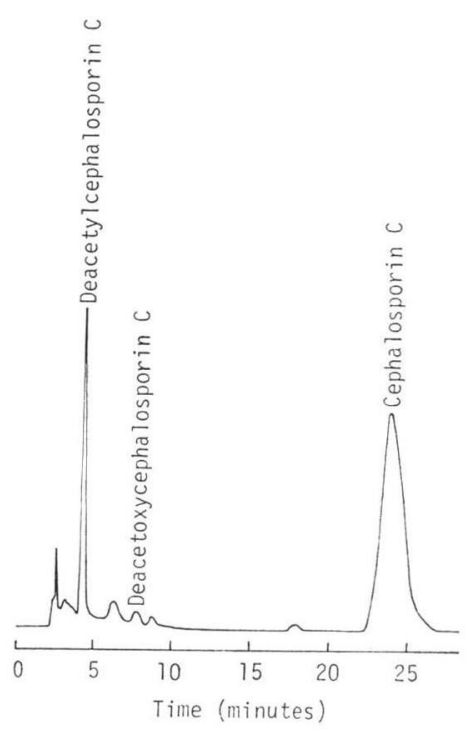

poor reverse phase column $\left(\mathrm{N}_{\mathrm{after}} / \mathrm{N}_{\text {before }}=0.5\right)$. The separation of a synthetic mix of cephalosporin $\mathrm{C}$ derivatives on this column is shown in Fig. 9.

Columns prepared from SI 60 silica and the difunctional bonding agent MODDCS were adequate from an efficiency standpoint, but were deficient in terms of peak tailing. As indicated by the $\mathrm{A}_{\mathrm{s}}$ values in the table and also in Figs. 10 and 11, separations are good on the MODDCS, SI 60 and MODDCS + TMS, SI 60 columns, but peak tailing is observed for all three cephalosporins.

The best results were obtained with methyloctadecyldichlorosilane and SI 100 silica. This is in agreement with the work of KARCH et al. ${ }^{7)}$ and independently with the work of HEMETSBERGER et al. ${ }^{3)}$ Both groups were able to prepare excellent packings from SI 100 silica and methyloctadecyldichlorosilane. Fig. 12 shows the separation obtained on the MODDCS, SI 100 column which is excellent in every respect. The efficiency of the column is high (1,300 plates), the $\mathrm{k}^{\prime}$ values are large enough and the peak shapes are excellent. These facts are also evident from the data given in Table 2. Fig. 13 shows the results of endcapping. The efficiency is still high (1,400 plates), the peak shapes are good and the $\mathrm{k}^{\prime}$ values, as expected, are somewhat larger. Note that deacetylcephalosporin $\mathrm{C}$ is very well separated from the solvent front $\left(\mathrm{k}^{\prime}=0.8\right)$, which makes quantitation possible without the need for ion-pairing.

Instead of just one custom made $\mathrm{C} 18$ column for the hydrophobic chromatography of cephalosporins, there are actually two that are suitable. Endcapping is optional, since both MODDCS, SI 100 and the MODDCS + TMS, SI 100 columns give high efficiencies, reasonable $\mathrm{k}^{\prime}$ values and symmetrical peaks. The separation of deacetylcephalosporin $\mathrm{C}$ is somewhat better on the endcapped (MODDCS + 
TMS) column.

\section{Conclusions}

The results given in this paper illustrate that custom made $\mathrm{C} 18$ columns prepared by an in-situ bonding technique are very useful for the analysis of cephalosporin $\mathrm{C}$ derivatives. These columns, which are prepared from $10 \mu \mathrm{m}, 100 \AA$ silica and methyloctadecyldichlorosilane, have been used routinely for the analysis of cephalosporin $\mathrm{C}$ in fermentation broths for a period of over five years.

\section{References}

1) White, E. R.; M. A. CARroll, J. E. Zarembo \& A. D. Bender: Reverse phase high speed liquid chromatography of antibiotics. J. Antibiotics 28: 205 214, 1975

2) White, E. R.; M. A. Carroll \& J. E. Zarembo: Reverse phase high speed liquid chromatography of antibiotics. II. Use of high efficiency small particle columns. J. Antibiotics 30: 811 818, 1977

3) White, E. R. \& J. E. ZaRembo: Reverse phase high speed liquid chromatography of antibiotics. III. Use of ultra high performance columns and ion-pairing techniques. J. Antibiotics 34: 836 844, 1981

4) KenNEDY,J.H.: High performance liquid chromatographic analysis of fermentation broths: Cephalosporin C and tylosin. J. Chromatog. Sci. 16: 492 495, 1978

5) CASSIDY, R. M.; D. S. LeGAY \& R. W. FreI: Study of packing techniques for small particle silica gels in high speed liquid chromatography. Anal. Chem. 46: 340 344, 1974

6) Gilpin, R. K.; D. J. CAmillo \& C. A. JANicKi: Preparation and use of in situ chemically bonded small particle silica as packings in high pressure liquid chromatography. J. Chromatogr. 121: 13 22, 1976

7) Karch, K.; I. Sebestian \& I. Halasz: Preparation and properties of reversed phases. J. Chromatogr. 122: $3 \sim 16,1976$

8) Hemetsberger, H.; M. Kellerman \& H. Ricken: Behavior of chemically bonded alkylmethyldichlorosilanes to silica gel in reversed-phase high performance liquid chromatography. Chromatographia 10: 726 730, 1977 